routinely applied 48 hours before the operation, according to the guidelines published by health authorities since June 2021 . Conclusion* There is no 'one size fits all' approach to cancer treatment during the COVID-19 pandemic, and there are no international guidelines. Screening and treatment decisions should often be made on a case-by-case basis and often depend on the COVID-19 situation in a single community and the availability of resources. Our study results shows that it can be done safely, even in the pandemic, when strict adherence to Covid 19 precautions for both patients and healthcare workers .

\section{DOWNREGULATING KIF4A SIGNIFICANTLY SUPPRESSED GROWTH OF UTERINE LEIOMYOSARCOMA}

C Mizuta*, S Nakagawa, K Hiramatsu, A Miyoshi, E Kobayashi, T Kimura, Y Ueda, T Kimura. Osaka University, Suita, Japan

\subsection{6/ijgc-2021-ESG0.263}

Introduction/Background* Uterine leiomyosarcoma (LMS) is notorious for its poor prognosis. New therapeutics strategy for LMS is mandatory. Kinesin family member4A (KIF4A) is a member of the kinesin 4 subfamily and plays an important role in cell division. In recent years, KIF4A is revealed to plays significant roles in some cancers with tumor proliferation. The aim of this study is to investigate the role of KIF4A in LMS. Methodology To identify novel biomarkers of LMS, we performed shotgun proteomics of one normal human uterine smooth muscle cell line (UtSMC) and three LMS cell lines, SK-LMS, SKN and SK-UT1, using isobaric tags for relative and absolute quantitation (iTRAQ). Cell variability was evaluated by MST- 8 assay in original LMS cells and KIF4A suppressed cells with siRNA in vitro. For evaluation of proliferation in vivo, we established KIF4A knockdown cell lines using shRNA and injected them subcutaneously to 6weeks ICR nude mice and evaluated changes in tumor size over time. To elucidate the mechanism, we performed cell cycle analysis by fluorescence-activated cell sorting (FACS) and western blotting.

Result(s)* A total of 2084 proteins were identified using iTRAQ. KIF4A was identified as a protein with more than twice the expression level of normal smooth muscle cells. By western blotting, all three LMS cell line had expressed KIF4A. KIF4A downregulation significantly suppressed the growth of those cell lines in vitro $(-37.2 \pm 4.73 \%$ in SK-LMS, -87.7 $\pm 4.02 \%$ in SKN and $-28.1 \pm 3.00 \%$ in SK-UT1, $\mathrm{p}<0.05)$. By FACS, the percentage of $\mathrm{G} 2 / \mathrm{M}$ phase cells was significantly

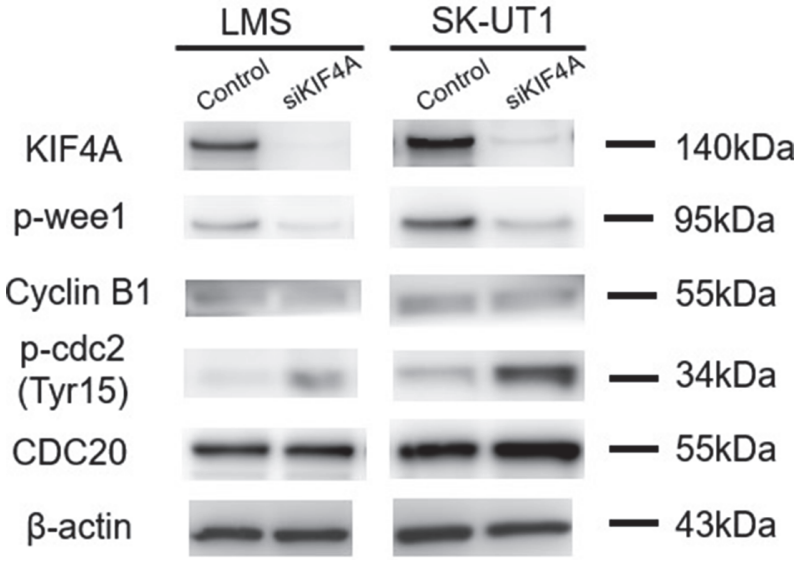

Abstract 379 Figure 1 The expression of proteinsinvolved in G2/M checkpoint

increased in KIF4A suppressed SK-LMS and SK-UT1 and by western blotting, p-cdc2 was upregulated in KIF4A suppressed cells (figure 1). It was suggested that KIF4A downregulation induced $\mathrm{G} 2 / \mathrm{M}$ arrest by inhibiting dephosphorylation of cdc2. Also in vivo, downregulation of KIF4A was found to significantly suppress tumor size $\left(1186 \pm 118 \mathrm{~mm}^{3}\right.$ vs $461 \pm 84 \mathrm{~mm}^{3}$ in SK-LMS and $1704 \pm 441 \mathrm{~mm}^{3}$ vs $514 \pm 230 \mathrm{~mm}^{3}$ in SK-UT1, $\mathrm{p}<0.05$, figure 2 ).

Conclusion* We identified a novel expressed protein, KIF4A, which can be a therapeutic target for uterine leiomyosarcoma.

\section{PULMONARY EMBOLISM IN GYNECOLOGIC ONCOLOGY}

1:2p Giannakas*, 'CR lavazzo, 'D Korfias, 'E Karavioti, 'D Giannoulopoulos, 'A Fotiou, 'S Lekka, 'K Kokkali, 'E Tepetzikiotis, ' $\mathrm{G}$ Vorgias. 'Metaxa Cancer Hospital of Piraeus, Gynecologic Department, PIRAEUS, Greece; '2Metaxa Cancer Hospital of Piraeus, Gynecologic Oncology Department, Piraeus, Greece

\subsection{6/ijgc-2021-ESG0.264}

Introduction/Background* All surgeons are in concern for thromboembolic events especially following a major operation for cancer. The aim of this audit is to report the incidence of $\mathrm{PE}$ in our oncology department, to identify risk factors and help us design future studies in order to reduce the incidence of PE and provide the best healthcare services in our patients. Methodology The Metaxa anti-cancer hospital database was reviewed to identify patients who had surgery performed for gynecologic malignancy from March 2019 to March 2021.
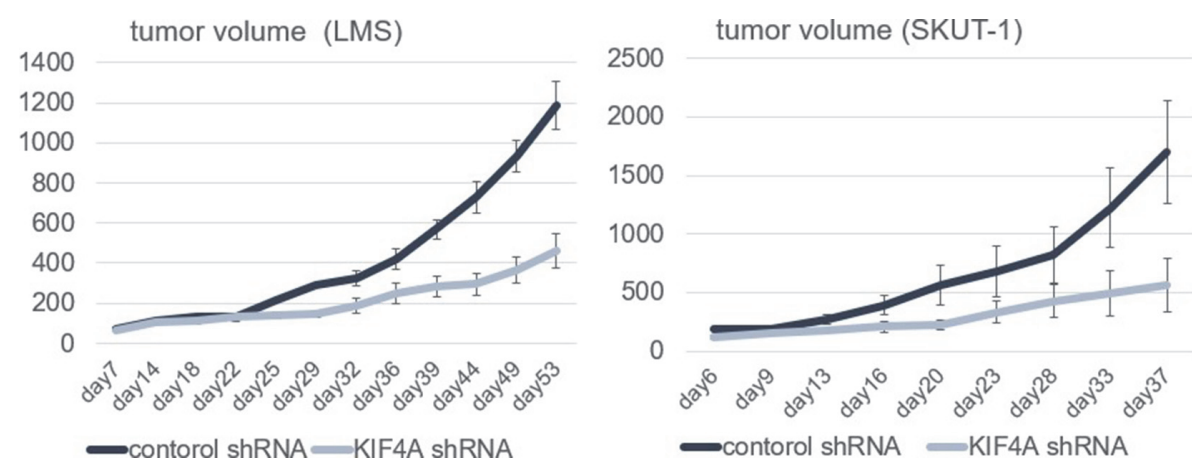

Abstract 379 Figure 2 The effect of suppressing growth in vivo 